La radiodifusión durante el conflicto del Atlántico Sur en 1982

Omar J. Somma - Arnaldo Leonel Slaen

Question/Cuestión, Vol. 2, №66, Agosto 2020

ISSNe 1669-6581

https://perio.unlp.edu.ar/ojs/index.php/question/index

IICom-FPyCS-UNLP

\title{
La radiodifusión durante el conflicto del Atlántico Sur en 1982
}

\section{Broadcasting during the South Atlantic conflict in 1982}

\section{Omar J. Somma y Arnaldo Leonel Slaen}

\section{Palabras clave}

Radiodifusión, Malvinas, Soberanía

\section{Keywords}

Broadcasting, Falklands, Sovereignty

\section{Introducción}

Durante este año 2020 celebramos el centenario de la radio en la República Argentina. Un acontecimiento de tanta significatividad puede ser abordado desde diferentes ópticas ya que muchos fueron los hitos que marcaron estos 
cien años de la radio en nuestro país y hay eventos que se han instalado a fuego en la memoria colectiva.

Es nuestra intención abordar la celebración desde un hecho muy especial y que ha permitido que la radio ocupe un papel preponderante como lo fue la guerra que en 1982 nos enfrentó con Gran Bretaña a fin de consolidar la recuperación de nuestras Islas Malvinas que se logró el 2 de Abril de ese año.

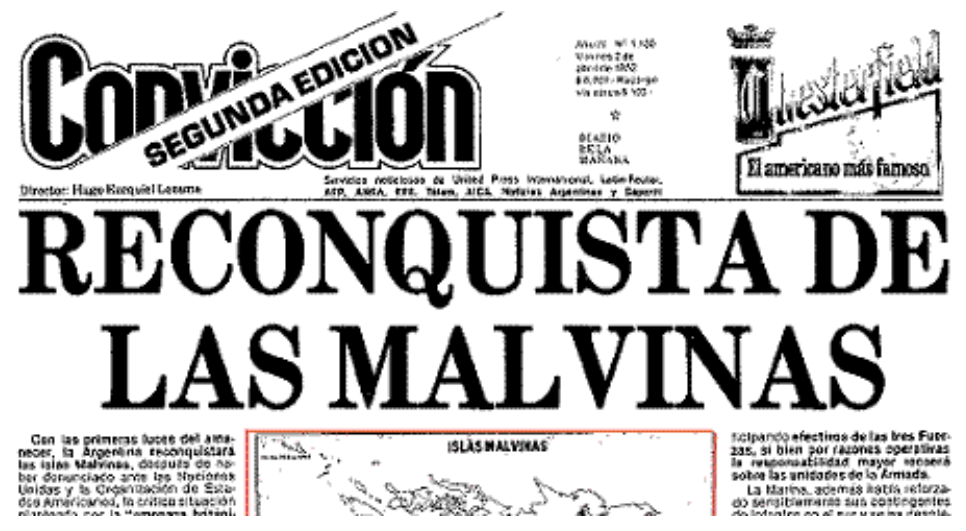

Así, el conflicto de las Islas Malvinas nos ha mostrado el amplio campo en el cual se desarrolla una guerra: el militar, el diplomático, el económico y, por supuesto, el de las comunicaciones. Nos adentraremos entonces en este ámbito, poniendo énfasis concretamente en el empleo de la radio como medio propagandístico durante la gesta por nuestras irredentas tierras australes.

En este aspecto, la mayoría de los países a lo largo del tiempo han reconocido las grandes posibilidades de la radio como arma eficaz tanto en épocas de guerra como de paz.

Históricamente, Alemania fue el primer país que supo utilizar este medio con fines netamente políticos. Durante la Segunda Guerra Mundial las 
transmisiones de Radio Tokyo, Radio Alemania, la BBC de Londres y otras estaciones fueron utilizadas con netos objetivos propagandísticos y militares por las potencias y países en conflicto.

El período de posguerra y la ulterior Guerra Fría trajo consigo la singular "guerra de las ondas". Durante estos años surgen infinidad de emisoras con particulares estilos propagandísticos a favor de sistemas sociales, políticos y económicos opuestos. Un ejemplo de ello son las emisiones de Radio Moscú, La Voz de los Estados Unidos o Radio Pekín. Junto con estas emisoras surgen otras de claro carácter político como lo son Radio Europa Libre y Radio Libertad, que emiten programas para los países del Este europeo y para las repúblicas que obtuvieron su independencia luego del desmembramiento y posterior desaparición de la Unión Soviética. Dentro de este grupo también se ubican las llamadas emisoras clandestinas que por lo general emiten de forma oculta en las propias zonas de conflicto o desde regiones cercanas 0 empleando sitios transmisores alquilados.

Esta introducción que es básicamente enumerativa y no taxativa nos sirve como una herramienta para entender el panorama radial que se presentó apenas iniciado el conflicto bélico.

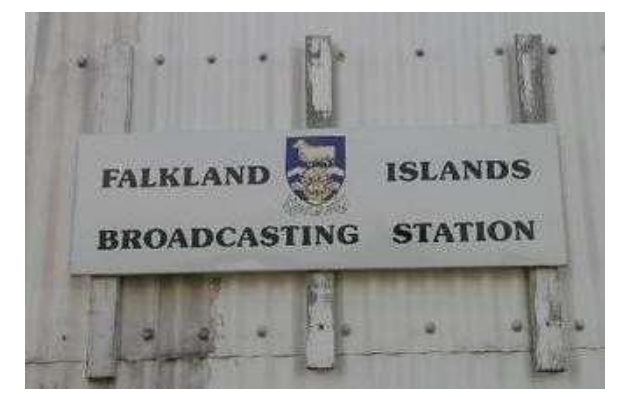




\section{Estado de situación}

Las Islas Malvinas contaban antes del 02 de Abril de 1982 con una estación de radio: el Servicio de Radio de las Islas Falklands (FIBS).- Las transmisiones eran en inglés y la estación contaba con programación local y servicios noticiosos tomados de la BBC de Londres. Trasnmitía en 536 khz y 2370 khz Con la frecuencia de amplitud modulada se pretendía cubrir la por entonces denominada Port Stanley y a través de la onda corta se buscaba alcanzar zonas más remotas de las islas.

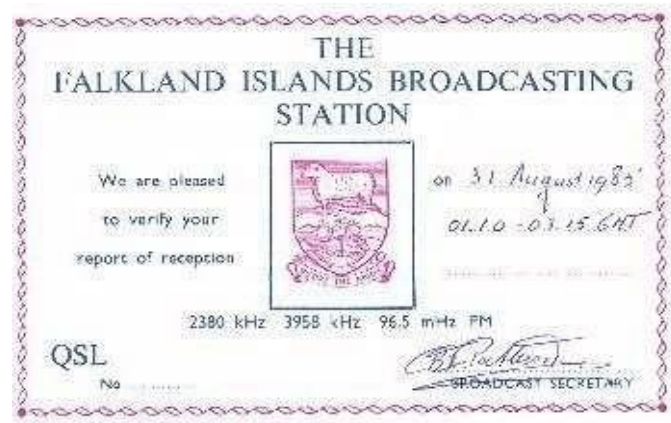

Al archipiélago iban también dirigidas las emisiones de Radiodifusión Argentina al Exterior (RAE) y de Radio Noticias Argentinas en idioma inglés, al igual que un servicio especial de la BBC de Londres dirigido a los "kelpers". Todas estas transmisiones se irradiaban por onda corta.

En cuanto a la televisión, sólo se recepcionaba en el archipiélago en algunas ocasiones el Canal 9 de Río Gallegos. 


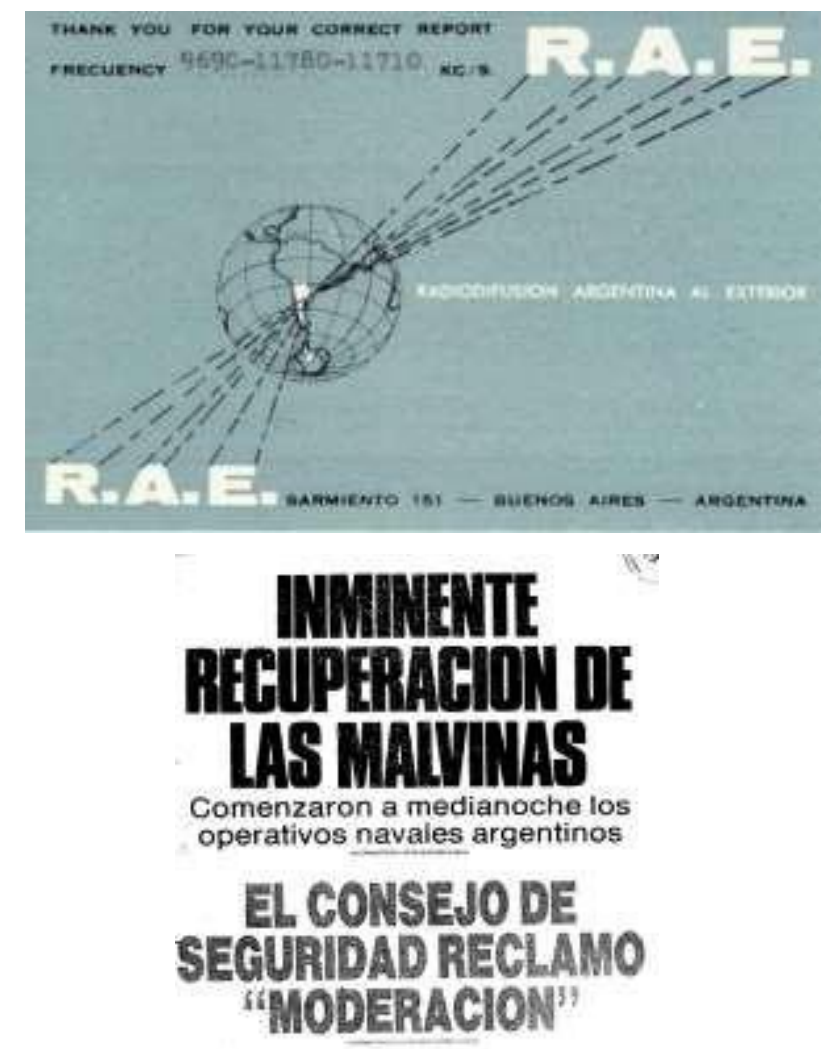

A partir del 02 de Abril de 1982, cuando nuestras Fuerzas Armadas recuperaron las Islas Malvinas y demás territorios insulares, la situación radial cambió drásticamente.

La FIBS fue ocupada militarmente y sus instalaciones fueron empleadas por la emisora que el Estado argentino integró a Radio Nacional puesto que por esos días comenzó a irradiar sus programas LRA60 Radio Nacional Islas Malvinas.

\section{El Conflicto}

Pero revisemos lo que sucedía en las horas previas a la toma de las islas, cuando la FIBS contaba exclusivamente con dos empleados permanentes y varios colaboradores a tiempo parcial que trabajaban conduciendo diferentes 
espacios de la emisora. El día anterior al de la recuperación de las islas, uno de sus locutores fijos, Patrick Watts, anunció a los habitantes el comienzo de las acciones militares de recuperación del archipiélago por parte de las fuerzas argentinas y la evolución posterior de los hechos que, durante toda la noche del 1 de abril y madrugada del 2 de abril, condujeron a la rendición incruenta de la guarnición militar inglesa y a la renuncia del gobernador.

Días posteriores a la recuperación exitosa de las Malvinas por parte de las tropas argentinas, nace LRA60 Radio Nacional Islas Malvinas. Esta icónica emisora contó entonces con la rápida incorporación de varios argentinos entre los que estaban Ernesto Manuel Dalmau, quien era ingeniero de sonido, Fernando Héctor Péndola que era operador y Norman Carlos Powell, quien era locutor oficial de LRA.

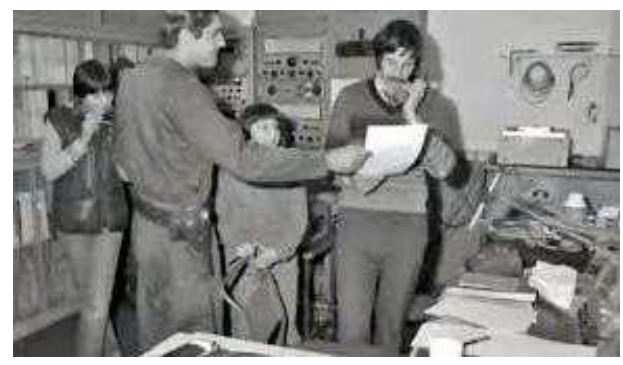

LRA60 utilizó los equipos e infraestructura de radiodifusión existentes y que pertenecían a la ya mencionadaa Falkland Island Broadcasting Station. (FIBS) No queremos dejar de mencionar que en esas jornadas durante las cuales nuestro país dio diferentes pasos en aras de consolidar la recuperación de las Islas, comenzó a transmitir LU78 Canal 7 de Televisión para dar servicio de radio y TV tanto para el personal militar y civil desplegado luego de la toma como para los habitantes de las islas. Ambas estaciones, LRA60 y LU78, 
transmitieron desde Puerto Argentino, que fue el nombre con el cual se denominó a la capital de las Malvinas (ex Port Stanley).

Patrick Watts se mantuvo trabajando en LRA60 a fin de brindar diversas informaciones e instrucciones a los habitantes de las Islas tanto en español como en inglés pero bajo el estricto control y respondiendo órdenes de las autoridades argentinas.

Durante el desarrollo de las hostilidades, Watts continuó siendo locutor de LRA60 para aprovechar su conocimiento del medio local y su manejo fluído del idioma inglés con todos los regionalismos empleados en Malvinas lo cual se convirtió en un grueso error de cálculo puesto que delató datos estratégicos militares a los británicos, diciendo al aire, por ejemplo, que el aeropuerto de las islas continuaba operativo a pesar de los bombardeos de los militares ingleses, en un claro intento encubierto de informar la situación a la flota de ataque enviada por el Reino Unido.

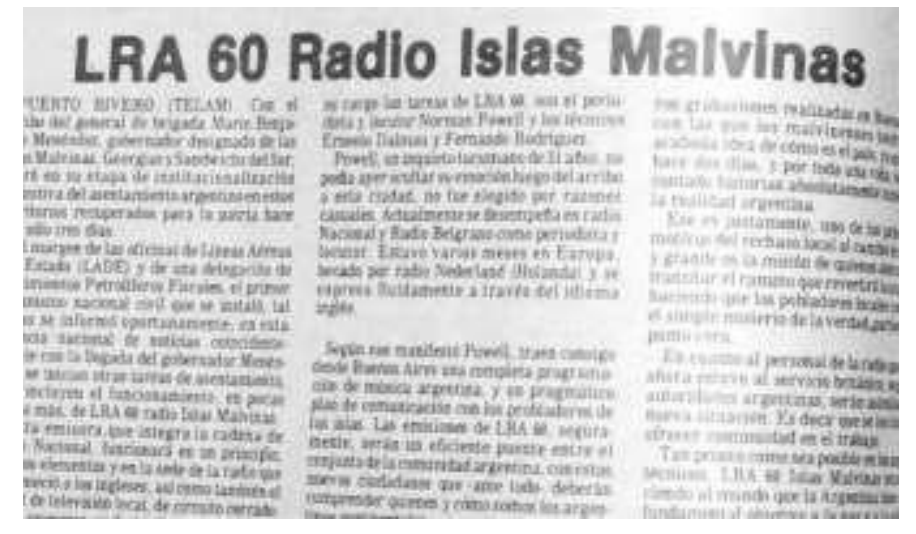

Durante el conflicto bélico y hasta la finalización del mismo, LRA60 Radio Nacional Islas Malvinas transmitió su programación por un sistema de cable emplazado dentro del perímetro de Puerto Argentino que se sumaba al empleo 
de su frecuencia de onda media en $536 \mathrm{KHz}$ y a la onda corta de $2370 \mathrm{KHz}$. La programación se retransmitió además en otras frecuencias de onda corta que fueron utilizadas como relé con la Argentina continental y de esta manera su programación fue recibida y retransmitida por otras emisoras argentinas. En varias ocasiones LRA60 fue cabecera de toda la red de emisoras del ya desaparecido Servicio Oficial de Radiodifusión.

Las frecuencias utilizadas como relé fueron las de $15890 \mathrm{KHz}$ y $24146 \mathrm{KHz}$ y correspondían a la estación de radio indicativo VPC instalada por la empresa Cable \& Wireless Co. que operaba en banda lateral única (USB ) y que las islas utilizaban para enlace con Inglaterra en comunicaciones punto a punto antes del estallido del conflicto.

La programación de LRA60 era muy variada e incluía música en inglés y en español, espacios de deportes retransmitidos directamente desde la BBC lo cual no deja de ser algo muy curioso y anuncios comunitarios destinados a la población urbana y rural en los dos idiomas. Se continuó también con la emisión habitual de los mensajes dirigidos a los pobladores rurales distribuidos en las diferentes estancias y granjas que están diseminadas en el archipiélago. Por esos ajetreados días, la República Argentina intensificó sus emisiones destinadas a las Malvinas. Se destinaban transmisiones especiales de RAERadiodifusión Argentina al Exterior que se irradiaban por la onda corta, a través de la frecuencia de $6060 \mathrm{KHz}$ y algunos programas generados en LRA11 Radio Nacional Comodoro Rivadavia. LRA24 Radio Nacional Rio Grande, desde la ciudad homónima, en la Provincia de Tierra del Fuego también contribuyó con la generación y envió de contenidos para LRA60.

Es interesante destacar que la señal de onda media de LRA60 también fue utilizado para anunciar y coordinar con la población rural de las dos islas más 
importantes del archipiélago (Gran Malvina y Soledad) los vuelos que se efectuaban hacia las mismas a fin de hacerles llegar correo y medicamentos con aterrizajes que se realizaban allí donde los mismos eran posible (a salvo de los furiosos bombardeos ingleses o de los ataques de la aviación colonial) o programando el lanzamiento de las cargas con dichos envíos desde el aire.

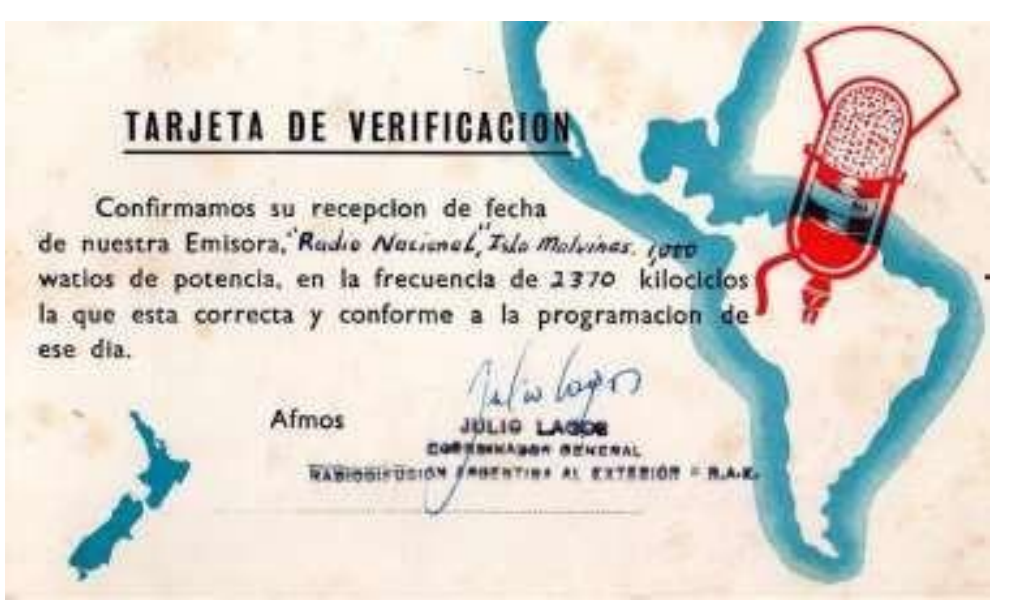

Sin embargo, en días posteriores al 1 de mayo de 1982, frecuentemente las señales de onda media y onda corta fueron silenciadas por los operadores de la radio ya que tenían buena recepción en Chile y eran utilizadas por escuchas del país trasandino con fines de espionaje en contra de la República Argentina. Estas transmisiones radiales volvieron a funcionar en forma intermitente, muchas veces sin programación, probablemente para ser utilizados como radiofaros por los aviones argentinos y solo volvieron a transmitir la programación de LRA60 durante la visita del Papa Juan Pablo II a la Argentina casi al final del conflicto. LRA60 continúo emitiendo por el sistema de cable dentro de Puerto Argentino. 
Pocos días antes del 14 de junio, fecha en que las fuerzas coloniales recuperaron las islas para Gran Bretaña, LRA60 Radio Nacional Islas Malvinas salió del aire debido a un corte en el suministro de energía eléctrica el que fue dañado por los intensos bombardeos británicos a Puerto Argentino. Esos mismos ataques aéreos y de artillerías naval y de las fuerzas de tierra también destruyeron la antena de onda media y su transmisor.

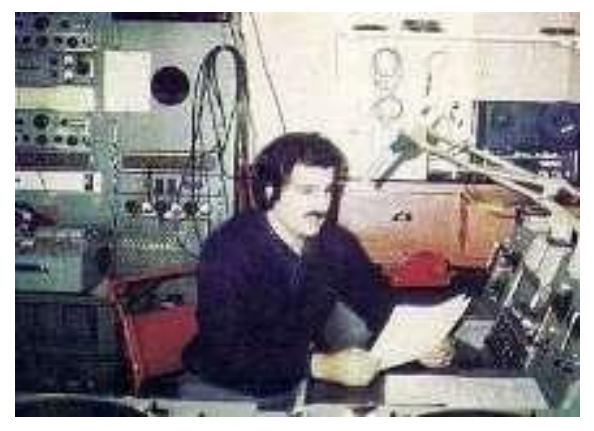

Pero retrocedamos a los primeros días de la recuperación de Malvinas nuevamente. Cuando Gran Bretaña anunció el envío de su gigantesca "Task Force" con el objetivo de volver a tomar las Islas, la República Argentina comenzó a operar una emisora propagandística cuyo nombre e historia nos remite a aquellos días del conflicto. Se trató de "Liberty", que se dirigía en idioma inglés a los soldados británicos que por aquel entonces comenzaban a cruzar el Océano Atlántico distribuidos en las decenas de barcos de guerra y buques de apoyo. "Liberty" transmitía en onda corta , a través de la frecuencia de $17.740 \mathrm{khz}$, en la banda de 16 metros y su objetivo era mostrarles a los soldados y marinos el inútil sacrificio que los miembros de la Royal Navy iba a hacer por un territorio lejano y desconocido para ellos.. 
"Liberty" era una dulce y sensual voz femenina que les hablaba a los jóvenes militares ingleses sobre la agresión que la Gran Bretaña realizaba a la Argentina. Ella, porque "Liberty" era esa mujer que hablaba a los oídos de los ingleses, les sugería y preguntaba si "no quisieran estar en casa y ver un partido de fútbol como aplaudir a sus favoritos....." mientras que les decía a todos y a cada uno de sus escuchas que “........estoy aquí para hacerles compañía..haz escrito a tu novia.....se que te mueres de ganas de verla..."

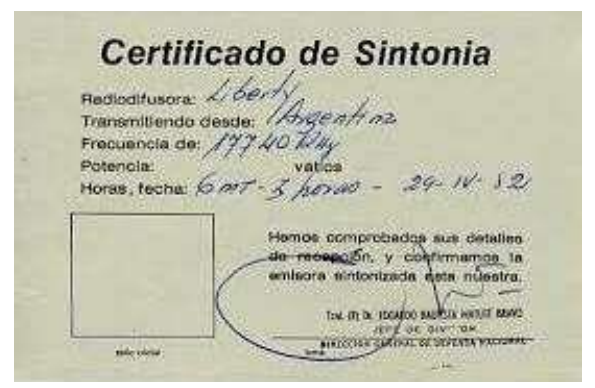

Radio Liberty o, simplemente Liberty, fue una genial creación radiofónica. Se presentaba cada día a través de su locutora, que anunciaba que su voz se podía escuchar en una hermosa casa en Belgrave Square o en un barco navegando en altamar para, saludar luego anunciado: "Hola soy Liberty, he decidido mostrarme al mundo desde un lugar que está muy lejos de usted, en Malvinas, Sandwich y Georgias del Sur, soy una voz, un espíritu, un país" mientras el tema "Yesterday", de The Beatles, sonaba de fondo.

Muchos años después de finalizado el conflicto bélico se supo que esa voz correspondía a Silvia Fernández Barrios, que por ese entonces era locutora de Argentina Televisora Color (LS82 TV Canal) mientras que en los contenidos colaboraba el conocido locutor y periodista Enrique Alejandro Mancini. En algunas ocasiones, a pesar del secreto, la emisión de Liberty se transmitía por 
Radio del Plata, los sábados por la noche. Tras hacer escuchar las campanas del Big Ben, se daban los resultados del fútbol inglés y se les recordaba la los soldados lo lejos que estaban de sus hogares. "Nos hemos encontrado, soy Liberty y tú eres Tomy, simpatizante del Tottenham, sí, debes estar navegando en alta mar, por eso hemos decidido brindarte compañía. ¿Te gustaría que te recuerde tu pueblo?", leía Fernández Barrio.

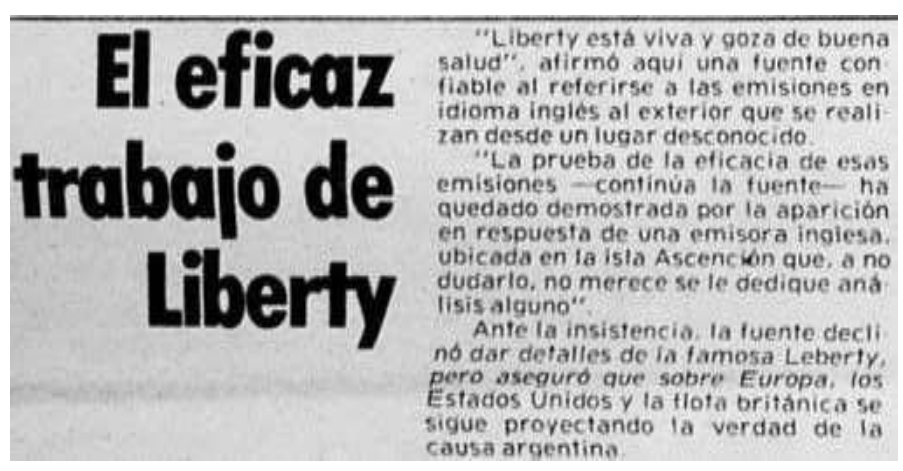

La misión básica de Liberty durante el enfrentamiento bélico en Malvinas fue muy claro: desalentar a los soldados ingleses que viajaban hacia nuestras Islas y luego cuando éstos comienzan a reocupar las mismas mediante mensajes nostálgicos que -tenía la ilusión- ayudarían a evitar o a disolver el conflicto en el territorio.

La Argentina también desata una ofensiva radiofónica por onda corta a través de Radiodifusión Argentina al Exterior que, en ocho idiomas (español, inglés, francés, árabe, portugués, alemán, italiano y japonés) trata de imponer el punto de vista del gobierno militar de entonces sobre la causa de Malvinas y sobre la situación interna de nuestro país y su posición internacional.

Finalmente, las autoridades militares argentinas de entonces pretendieron atender las necesidades de entretenimiento de los soldados argentinos 
acantonados en las Islas Malvinas y en diferentes puntos geográficos de la Patagonia a través de la retransmisión en diferentes frecuencias de onda corta de tres de las emisoras más populares de entonces: LS10 Radio del Plata (muy apreciada entre los jóvenes por sus propuestas musicales); LS5 Radio Rivadavia (cuyos programas deportivas y transmisiones de fútbol, boxeo y automovilismo eran seguidas por millones de compatriotas) y LS4 Radio Continental (con una gran audiencia por sus contenidos periodísticos).También por esos días estaban operativas en sus frecuencias habituales de onda corta otras tres prestigiosas emisoras de Buenos Aires: LR3 Radio Belgrano, LR4 Radio Splendid y LR1 Radio El Mundo. Las frecuencias empleadas por estas estaciones con mucha historia en el medio local estaba distribuidas en bandas de 49, 31, 25 y 19 metros. Es indispensable señalar que todas estas radios, salvo excepciones, estaban intervenidas por los militares desde el año 1976 y su programación era rigurosamente censurada.

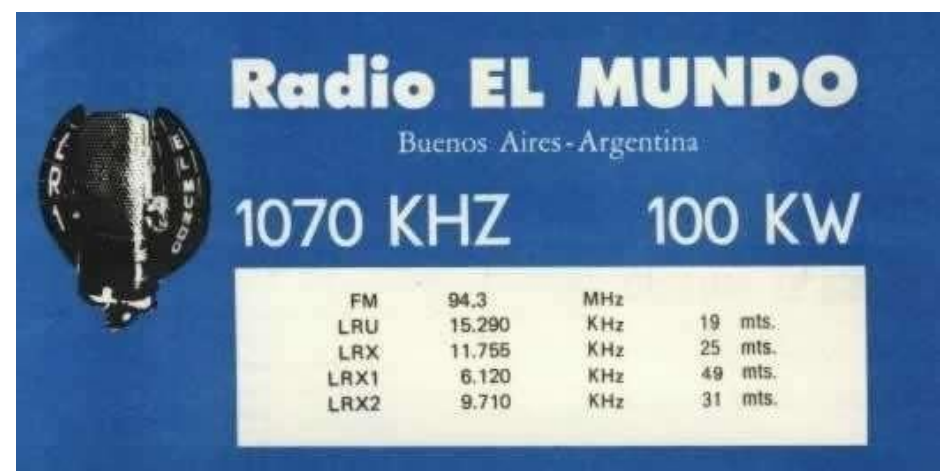

Este informe sería incompleto si no señalásemos que en el aspecto internacional, todas las grandes emisoras internacionales del mundo dedicaron largos espacios a los acontecimientos del Atlántico Sur. 


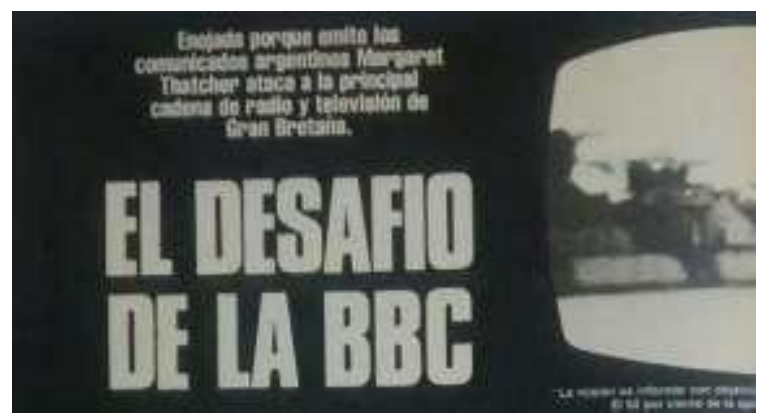

La BBC aumentó durante la Guerra de Malvinas la duración de las transmisiones de su Servicio en Español para Latinoamérica al igual que sus emisiones en inglés dirigidas a los pobladores de las Islas. A partir del 01 de Mayo de 1982 esta guerra del éter se intensificó: mientras que la BBC denunciaba que las emisiones de su Servicio Latinoamericano eran interferidas desde Argentina, las autoridades de Radio Nacional de Buenos Aires comunicaban que sus servicios de ondas cortas eran interferidas, posiblemente, desde la zona norte del continente. americano.

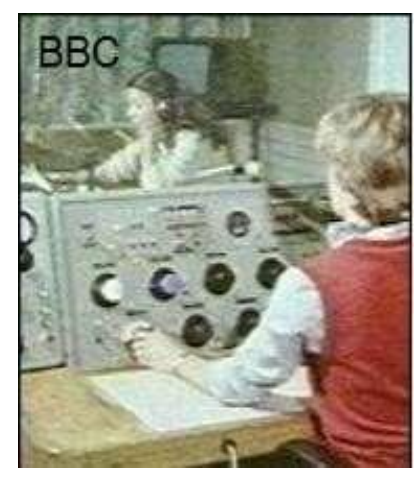

El Ministerio de Defensa británico, descontento con la forma en que la BBC manejaba la información de guerra, se hizo cargo de uno de los transmisores de esta emisora emplazado en la isla de Ascensión, en el Océano Atlántico, cerca de las costas de Africa e inició, el 19 de mayo de 1982 emisiones en idioma español bajo el nombre de Radio Atlántico del Sur con el fin de, según 
sus propias palabras, "llevar calor a los aislados soldados argentinos...... dándoles a conocer la verdad nacional e internacional..."Esta estación transmitía hacia nuestros soldados instalados en las islas empleando la frecuencia de $9710 \mathrm{khz}$, en la banda de 31 metros.

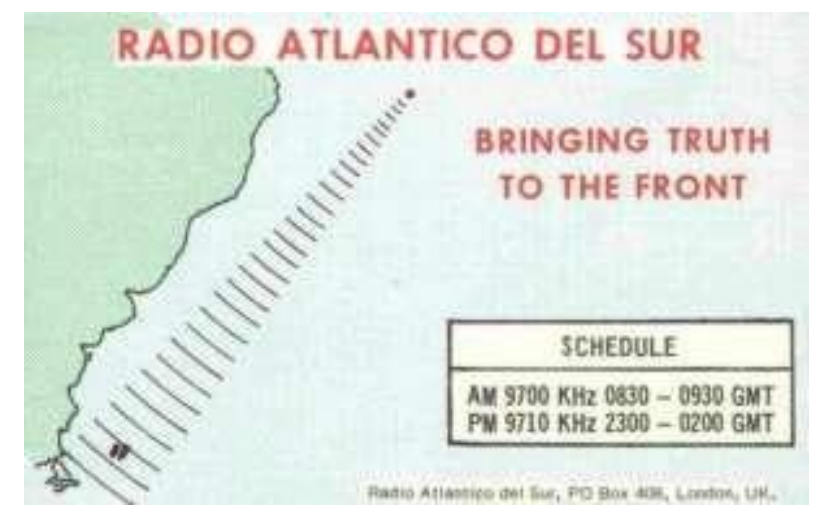

Salía al aire con una variada programación en español producida en Londres, que incluía desde espacios informativos (incluyendo noticias de las ciudades de origen de los soldados), resultados de partidos de fútbol, mensajes presuntamente escritos por madres de soldados argentinos para sus hijos, música popular «del agrado de los argentinos» e incluso una "pausa sentimental". El equipo de locutores estaba integrado tanto por británicos como por latinoamericanos, que carecían de una adecuada capacitación para transmisiones en vivo. En efecto, los conductores y locutores eran de diferentes países centroamericanos por lo cual los tonos y modos empleados no eran los acostumbrados para los soldados argentinos. Si bien fue una emisora de poco peso comunicacional siempre trató de ser la contracara británica de Radio Liberty. 


\section{Final}

En ocasión de conmemorar una fecha tan especial como lo es el centenario de la radio en la Argentina que se celebra este año no podemos dejar de recordar el escenario radiofónico durante la guerra disputada por nuestro país luego de la recuperación de las Islas Malvinas y hasta su finalización.

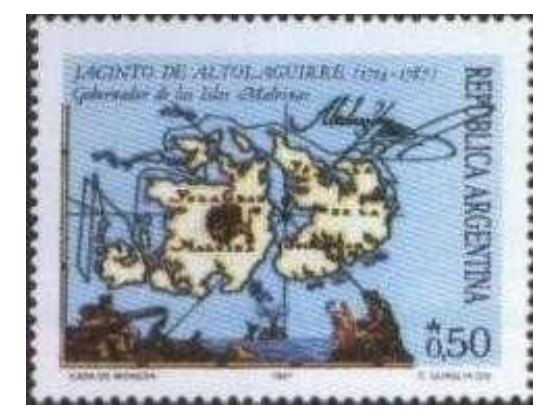

\section{Bibliografía}

- Manual Mundial de Radio \& Televisión (World Radio \& TV Handbook)

- Nota "Historia de LRA60 Radio Nacional Islas Malvinas", escrita por el Ingeniero Alejandro Daniel Alvarez

- Voces en las sombras "una historia de las radios clandestinas" cuyo autor es Luis Zaragoza

- La guerra de las ondas radiales, Omar J. Somma, Novedad e impacto $N^{\circ}$ 3, agosto 1982 